\title{
COMPLEX ITERATED RADICALS
}

\author{
LEON GERBER
}

\begin{abstract}
We prove the convergence of the sequence $S$ defined by $z_{n+1}=\left(z_{n}-c\right)^{1 / 2}, c$ real, for any choice of $z_{0}$. Let $k=\left|\frac{1}{4}-c\right|^{1 / 2}$. If $c<0$ or $c=\frac{1}{4}, S$ has only one fixed point $w=\frac{1}{2}+k$ and converges to $w$ for any $z_{0}$. If $0 \leqq c<\frac{1}{4}, S$ has the fixed points $w_{1}=\frac{1}{2}+k$ and $w_{2}=\frac{1}{2}-k$, and for any $z_{0} \neq w_{2}, S$ converges to $w_{1}$. If $c>\frac{1}{4}, S$ has the fixed points $w_{1}=\frac{1}{2}+i k$ and $w_{2}=\frac{1}{2}-i k$ and converges to $w_{1}$ if $\operatorname{Re}\left(z_{0}\right) \geqq 0$ and to $w_{2}$ otherwise. We show that convergence is strictly monotone when the neighborhood system is the pencil of coaxial circles with $w_{1}$ and $w_{2}$ as limiting points, and give rates of convergence.
\end{abstract}

The purpose of this paper is to prove that the sequence of complex numbers defined by $z_{n+1}=\left(z_{n}-c\right)^{1 / 2}, c$ real, converges for any choice of $z_{0}$, i.e. globally, and to discuss the limit and rate of convergence. This problem was posed by C. S. Ogilvy [1].

As a guide to global convergence we first investigate local convergence. The following facts are known about the sequence defined by $z_{n+1}=f\left(z_{n}\right)$ for some choice of $z_{0}$.

LEMMA 1. If $\lim _{n \rightarrow \infty} z_{n}=w$ and $f$ is continuous at $w$ then $f(w)=w$, i.e. $w$ is a fixed point.

Proof. $\quad w=\lim _{n \rightarrow \infty} z_{n+1}=\lim _{n \rightarrow \infty} f\left(z_{n}\right)=f\left(w^{\prime}\right)$.

Suppose $w$ is a fixed point of $f$. If $z_{N}=w$ for some $N$, then $z_{n}=w$ for all $n \geqq N$ and we call the sequence trivial. We define

$$
q(n, w)=\left|z_{n+1}-w\right| /\left|z_{n}-w\right| .
$$

LEMma 2. Suppose $w$ is a fixed point of $f$ and $f^{\prime}\left(w^{\prime}\right)$ exists. If $\left|f^{\prime}(w)\right|<1$, then for $z_{0}$ sufficiently close to $w$ the sequence converges to $w$ and for all nontrivial sequences $\lim _{n \rightarrow \infty} q(n, w)=\left|f^{\prime}(w)\right|$ and we say that $\left|f^{\prime}(w)\right|$ is the local rate of convergence. If $\left|f^{\prime}(w)\right|>1$, then the sequence cannot converge to $w$ except trivially.

Received by the editors June 15, 1972 and, in revised form, February 13, 1973.

AMS (MOS) subject classifications (1970). Primary 40A05, 41A25; Secondary 39A10, 50D45.

Key words and phrases. Iterated radicals, convergence, rate of convergence, coaxial circles.

(c) American Mathematical Society 1973 
Proof. The result for trivial sequences is immediate. Otherwise $q(n, w)=\left|z_{n+1}-w\right| /\left|z_{n}-w\right|=\left|f\left(z_{n}\right)-f(w)\right| /\left|z_{n}-w\right|$. If $\left|f^{\prime}(w)\right|=1-2 \varepsilon$ for some $\varepsilon>0$, then $q(n, w)<1-\varepsilon$ for $z_{n}$ in some deleted circular neighborhood $\mathscr{M}$ of $w$ by the definition of derivative. Since $\mathscr{M}$ is circular, $z_{n+1}$ also lies in $\mathscr{M}$. For $z_{0}$ in $\mathscr{M}$ it follows that $\left|z_{n}-w\right|<\left|z_{0}-w\right|(1-\varepsilon)^{n}$. The conclusion is immediate.

If $\left|f^{\prime}(w)\right|=1+2 \varepsilon$ for some $\varepsilon>0$ then $q(n, w)>1+\varepsilon$ for $z_{n}$ in some deleted neighborhood $\mathscr{M}$ of $w$ so that the sequence is not eventually in $\mathscr{M}$.

We now focus on the sequence defined by $z_{n+1}=\left(z_{n}-c\right)^{1 / 2}$ where the argument of the square root lies in $\left(-\frac{1}{2} \pi, \frac{1}{2} \pi\right]$. We note that $\operatorname{Re}\left(z_{n+1}\right) \geqq 0$ for $n \geqq 0$ and that if $w$ is a fixed point, $z_{N}=w$ implies $z_{N-1}=w$ and thus $z_{0}=w$ so we exclude the cases $\operatorname{Re}\left(z_{0}\right)<0$ and $z_{0}=w$ from further discussion. We define $k=\left|\frac{1}{4}-c\right|^{1 / 2}$.

Lemma 3. If $c<\frac{1}{4}$, the sequence converges locally to $w_{1}=\frac{1}{2}+k$ at the rate $(1+2 k)^{-1}$. For $0 \leqq c<\frac{1}{4}, w_{2}=\frac{1}{2}-k$ is the other fixed point but the sequence cannot converge to $w_{2}$.

If $c>\frac{1}{4}$, the sequence converges locally to $w_{1}=\frac{1}{2}+k i$ and $w_{2}=\frac{1}{2}-k i$ at the rate $\left(1+4 k^{2}\right)^{-1 / 2}$.

Proof. The roots of $w^{2}=w-c$ are $w_{1}+\left(\frac{1}{4}-c\right)^{1 / 2}$ and $w_{2}=\frac{1}{2}-\left(\frac{1}{4}-c\right)^{1 / 2}$ and these are the roots of $w=(w-c)^{1 / 2}$ except when $c<0$ in which case $w_{2}<0$. Since $f(z)=(z-c)^{1 / 2}, f^{\prime}(w)=\frac{1}{2}(w-c)^{1 / 2}=\frac{1}{2} w$.

If $c<\frac{1}{4}, f^{\prime}\left(w_{1}\right)=(1+2 k)^{-1}<1$.

If $0<c<\frac{1}{4}, f^{\prime}\left(w_{2}^{\prime}\right)=(1-2 k)^{-1}>1$.

If $c>\frac{1}{4},\left|f^{\prime}\left(w_{1}\right)\right|=\left|f^{\prime}\left(w_{2}^{\prime}\right)\right|=\frac{1}{2} c^{1 / 2}=\left(1+4 k^{2}\right)^{-1 / 2}<1$.

We shall now prove global convergence and obtain rates very nearly equal to the local rates of convergence. We first consider two simple cases.

ThEOREM 1. For $c<0$, the sequence converges to $w_{1}$ for all $z_{0}$ and $q\left(n, w_{1}\right) \leqq\left(\frac{1}{2}+k\right)^{-1}$. For $c>1$, the sequence converges to $w_{1}$ if $\operatorname{Im}\left(z_{0}\right) \geqq 0$ and to $w_{2}$ if $\operatorname{Im}\left(z_{0}\right)<0$. In each case $q(n, w) \leqq\left(\frac{1}{4}+k^{2}\right)^{-1 / 2}$.

Proof. For any $c$ and either $w$,

$$
1 / q(n, w)=\left|z_{n}-w^{\prime} /\right| z_{n+1}-w|=| z_{n+1}+w \mid .
$$

For $c<0, w_{1}=\frac{1}{2}+k$ is real and $\operatorname{Re}\left(z_{n+1}\right) \geqq 0$ so $\left|z_{n+1}+w_{1}\right| \geqq w_{1}$. For $c>\frac{1}{4}$ and a fortiori for $c>1, w_{1}=\frac{1}{2}+k i$ lies in the first quadrant and if $z_{0}$ lies in the closed first quadrant so does $z_{n}$ for each $n$ so

$$
\left|z_{n+1}+w_{1}\right| \geqq\left|w_{1}\right|=c^{1 / 2}=\left(\begin{array}{c}
1 \\
4
\end{array}+k^{2}\right)^{1 / 2} .
$$

The argument is similar if $\operatorname{Im}\left(z_{0}\right)<0$. 
The factor in the first case is twice the local rate; the factor in the second case will be improved in Theorem 3 .

If $\frac{1}{4}<c<1, z_{n}=c+2 \dot{\varepsilon}^{2} i$, and $\varepsilon>0$ is sufficiently small, then

$$
\left|z_{n+1}+w\right|=|\varepsilon+\varepsilon i+w|<|w|+2 \varepsilon=c^{1 / 2}+2 \varepsilon<1 .
$$

If $0<c<\frac{1}{4}$ and $c<z_{n}<w_{2}$, then $0<z_{n+1}<z_{n}$ and again $q(n, w)>1$. Since, as we shall prove, every sequence converges, we see that a global convergence factor cannot be expressed in terms of the usual metric in which the distance of $z_{n}$ from $w$ is the radius of the circle passing through $z_{n}$ with $w$ as center. Instead, for each value of $c$, we set up a metric based on pencils of coaxial circles with $w_{1}$ and $w_{2}$ as limiting points.

For $0 \leqq c \leqq \frac{1}{4}$ we have $w_{1}=\frac{1}{2}+k$ and $w_{2}=\frac{1}{2}-k$ where $0 \leqq k \leqq \frac{1}{2}$. The centers of these circles lie on the real axis outside the interval $\left(\frac{1}{2}-k\right.$, $\left.\frac{1}{2}+k\right)$ and the circle with center $\frac{1}{2} \pm H$ has radius $R$ where $R^{2}+k^{2}=H^{2}$. The line $x=\frac{1}{2}$ is the radical axis of the pencil. For $c=\frac{1}{4}, w_{1}=w_{2}=\frac{1}{2}$ and the circles are tangent to $x=\frac{1}{2}$ at $\left(\frac{1}{2}, 0\right)$. For each $z$ not on the radical axis there is precisely one circle $C(z)$ of the pencil passing through $z$. These are the only properties of the pencil we require. We define $d\left(z, w_{1}\right)$ to be the radius of $C(z)$ if $\operatorname{Re}(z)>\frac{1}{2}$ and $\infty$ otherwise, and $d\left(z, w_{2}\right)$ to be the radius of $C(z)$ if $\operatorname{Re}(z)<\frac{1}{2}$ and $\infty$ otherwise. Notice that $d(z, w)$ and $|z-w|$ are asymptotic for $z$ near $w$ if $c \neq \frac{1}{4}$.

THEOREM 2. For $0 \leqq c \leqq \frac{1}{4}, d\left(z_{n}, w_{2}^{\prime}\right)$ is a strictly increasing function of $n$ as long as it is finite and $d_{n}=d\left(z_{n}, w_{1}\right)$ is eventually finite. Then $d_{n}$ is strictly decreasing to zero. In fact

$$
\begin{array}{ll}
d_{n+1} \leqq\left(\frac{1}{2} d_{n}\right)^{1 / 2} & \text { for } d_{n} \geqq \frac{1}{2}, \\
1 / d_{n+1} \geqq 1 / d_{n}+1 & \text { for } d_{n} \leqq 1, \\
1 / d_{n+1} \geqq 1 / d_{n}+2-4 d_{n} & \text { for } d_{n} \leqq \frac{1}{4}, \text { and } \\
d_{n+1} \leqq(1+4 k)^{-1 / 2} d_{n} & \text { for } c<\frac{1}{4} \text { and all } d_{n}<\infty .
\end{array}
$$

Comments. For $c=\frac{1}{4}$ the next to the last inequality gives the correct asymptotic rate of convergence. For $0 \leqq c<\frac{1}{4}$ the global convergence factor of $(1+4 k)^{-1 / 2}$ is never more than $2 \cdot 3^{-1 / 2}=1.15$ times the local convergence factor $(1+2 k)^{-1}$. When we restrict $\operatorname{Re}\left(z_{n}\right) \geqq \frac{1}{2}$ the global factor for $c<0$ of Theorem 1 improves to $(1+k)^{-1}$.

Proof. Suppose $d\left(z_{n+1}, w_{2}\right)=R<\infty$ i.e.

$$
z_{n+1}=\frac{1}{2}-H+R \cos \theta+i R \sin \theta=u+i R \sin \theta
$$

where $H=\left(R^{2}+k^{2}\right)^{1 / 2}$ and $0 \leqq u<\frac{1}{2}$. Let $r=a R$ and $h=\left(r^{2}+k^{2}\right)^{1 / 2}$. We shall show for the proper choice of $a<1$ that $\left|z_{n}-\left(\frac{1}{2}-h\right)\right|<r$, i.e. $d\left(z_{n}, w_{2}\right)<r$. 
This implies that $d\left(z_{n+1}, w_{2}\right)>a^{-1} d\left(z_{n}, w_{2}\right)$. In fact

$$
\begin{aligned}
\left|z_{n}-\left(\frac{1}{2}-h\right)\right|^{2}-r^{2} & =\left|z_{n+1}^{2}+c-\frac{1}{2}+h\right|^{2}-r^{2} \\
& =\left|(u+i R \sin \theta)^{2}-k^{2}-\frac{1}{4}+h\right|^{2}-r^{2} \\
& =|(2 u R \cos \theta+h-H)+2 u R i \sin \theta|^{2}-r^{2},
\end{aligned}
$$

after substituting for $u^{2}$ and $k^{2}=H^{2}-R^{2}$,

$$
\begin{aligned}
& =(2 u R \cos \theta+h-H)^{2}+(2 u R \sin \theta)^{2}-r^{2} \\
& =4(R \cos \theta-H)\left[R^{2}\left(u+\frac{1}{2}\right)-(H-h)(u+H)\right],
\end{aligned}
$$

after substituting for $u^{2}$ again. The first factor is negative Since

$$
H-h=\frac{H^{2}-h^{2}}{H+h}=\frac{R^{2}-r^{2}}{H+h}=\frac{R^{2}\left(1-a^{2}\right)}{H+h},
$$

the second factor equals

$$
\begin{aligned}
R^{2}\left(u+\frac{1}{2}\right)- & \frac{R^{2}\left(1-a^{2}\right)(u+H)}{H+h} \\
& >R^{2}\left[u+\frac{1}{2}-\left(1-a^{2}\right)\left(u+\frac{3}{4}\right) / 2 k\right], \text { for } H \leqq \frac{3}{4}, \\
& >0 \text { for } a=(1-4 k / 3)^{1 / 2} .
\end{aligned}
$$

Thus for $0 \leqq c<\frac{1}{4}$ the relation $d\left(z_{n+1}, w_{2}\right)>(1-4 k / 3)^{-1 / 2} d\left(z_{n}, w_{2}\right)$ is valid until $H$ exceeds $\frac{3}{4}$. If $c=\frac{1}{4}$ then $H=R$ and $h=r=a R$ and the second factor rearranges to $R\left[(a+R-1) u+\left(a-\frac{1}{2}\right) R\right]$ which is positive for $a=\max \left(1-R, \frac{1}{2}\right)$. Here too $H$ eventually exceeds $\frac{3}{4}$. Now the preimage of the line $x=\frac{1}{2}$ under the map $f(z)=(z-c)^{1 / 2}$ is the parabola $x=c+\frac{1}{4}-y^{2}$ and the portion of this parabola in the right half-plane is interior to the circle corresponding to $H=\frac{3}{4}$, i.e. the circle with center $-\frac{1}{4}$ and radius $(c+5 / 16)^{1 / 2}$. Thus once an iterate has a value of $H$ exceeding $\frac{3}{4}$ it lies outside the parabola and the next iterate lies to the right of $x=\frac{1}{2}$. This proves the first assertion.

When $d\left(z_{n+1}, w_{1}\right)=R<\infty$ we need to show for the proper choice of $a>1$ that $\left|z_{n}-\left(\frac{1}{2}+h\right)\right|>r$. Changing the signs of $H$ and $h$ in (1) and rearranging we get

$$
\begin{aligned}
\mid z_{n} & -\left.\left(\frac{1}{2}+h\right)\right|^{2}-r^{2} \\
& =2(R \cos \theta+H)\left[2 R^{2}(R \cos \theta+H+1)-(h-H)(2 R \cos \theta+1)\right] .
\end{aligned}
$$

The first factor is positive and $R \cos \theta+H+1>2 R \cos \theta+1$ so all we require is $2 R^{2} \geqq h-H$. Now $\left(2 R^{2}+H\right)^{2}-h^{2}=R^{2}\left(4 R^{2}+4 H+1-a^{2}\right)$. Since $R \leqq H$ the expression is nonnegative for $a=2 R+1$ which implies $r=R(2 R+1)$ so $R<(r / 2)^{1 / 2}$. By the quadratic formula $1 / R \geqq\left[1+(1+8 r)^{1 / 2}\right] / 2 r$ which yields the next two results. We get the final result with $a^{2}=1+4 k<1+4 h$. 
If $c>\frac{1}{4}$ then $w_{1}=\frac{1}{2}+k i$ and $w_{2}=\frac{1}{2}-k i$. The centers of the circles of the pencil lie on $x=\frac{1}{2}$ and the circles with center $\frac{1}{2} \pm H i$ have radius $R$ where $R^{2}+k^{2}=H^{2}$. The real axis is the radical axis. For each $z$ not on the radical axis there is precisely one circle $C(z)$ of the pencil passing through $z$. We define $d\left(z, w_{1}\right)$ to be the radius of $C(z)$ if $\operatorname{Im}(z)>0$ and $\infty$ otherwise and $d\left(z, w_{2}\right)$ to be the radius of $C(z)$ if $\operatorname{Im}(z)<0$ and $\infty$ otherwise.

THEOREM 3. For $c>\frac{1}{4}$, if $z_{0}$ is real then $d\left(z_{n}, w_{1}\right)$ is eventually finite. In fact, for a fixed $z_{0}>\frac{1}{2}$ and $c$ near $\frac{1}{4}$, the number of iterations required is asymptotic to $\pi / k$. If $0<d\left(z_{n}, w_{i}\right)<\infty$, then

$$
d\left(z_{n+1}, w_{i}\right) \leqq\left(1+k^{2}\right)^{-1 / 2} d\left(z_{n}, w_{i}\right), \quad i=1,2 .
$$

PRoof. If $z_{n+1}$ is real, then $z_{n+1}=\left(z_{n}-c\right)^{1 / 2} \leqq\left(z_{n}-c\right)+\frac{1}{4}=z_{n}-\left(c-\frac{1}{4}\right)$. Thus some iterate is less than $c$ and the next lies in the first quadrant. If $z_{0}<2^{2^{m}}$ then clearly $z_{n}<2$ for some $n \leqq m$. To estimate the number $N$ of steps required for $z$ to move from 2 to $c$ for small values of $k$, observe that

$$
z_{n+1}-z_{n}=z_{n+1}-\left(z_{n+1}^{2}+c\right)=-\left(z_{n+1}-\frac{1}{2}\right)^{2}-\left(c-\frac{1}{4}\right),
$$

so if we let $z_{n}=\frac{1}{2}-k x_{n}, x$ ranges from $-3 / 2 k$ to $\left(\frac{1}{4}-k^{2}\right) / k$ and $x_{n+1}-$ $x_{n}=\left(x_{n+1}^{2}+1\right) k$. Let $p$ be a positive integer. If $x_{n} \geqq 2^{j} p$ then $x_{n+1}-x_{n}>$ $2^{2 j} p^{2} k$ so that the number of steps for $x$ to move from $2^{j} p$ to $2^{j+1} p$ is less than

$$
\left(2^{j+1} p-2^{j} p\right) / 2^{2 j} p^{2} k+1=1 / 2^{j} p k+1, \quad j=0,1, \cdots .
$$

Thus the total number of steps required to move from $p$ to $\left(\frac{1}{4}-k^{2}\right) / k$ is less than $2 / p k+\log _{2}\left[\left(\frac{1}{4}-k^{2}\right) / p k\right]<3 / p k$. A similar argument shows that the number of steps from $-3 / 2 k$ to $-p$ is also bounded by $3 / p k$. Now take $x_{0}=-p$ and $x_{M}=p$, i.e. $M$ is the number of steps from $-p$ to $p$. Then

$$
\sum_{n=0}^{M-1} \frac{x_{n+1}-x_{n}}{x_{n+1}^{2}+1}=M k
$$

The left side is a Riemann sum for $\int_{-p}^{p} d x /\left(x^{2}+1\right)$ and since $x_{n+1}-x_{n} \leqq$ $\left(p^{2}+1\right) k$, the norm approaches zero with $k$. Clearly,

$$
2 \tan ^{-1} p<\lim _{k \rightarrow 0} M k<2 \tan ^{-1} p+\frac{6}{p}<\pi+\frac{6}{p} .
$$

Since $p$ can be arbitrarily large we have the result. Computer calculations show that if $c=0.250001$ i.e. $k=0.001$ and $z_{0}=2$, then $z_{3139}<c<z_{3138}$.

Suppose now $d\left(z_{n+1}, w_{1}\right)=R<\infty$, i.e.

$$
z_{n+1}=\left(\frac{1}{2}+H i\right)+R \cos \theta+i R \sin \theta=\frac{1}{2}+R \cos \theta+i v,
$$


where $v=H+R \sin \theta>0$. We shall show that $d\left(z_{n}, w_{1}\right) \geqq\left(1+k^{2}\right)^{1 / 2} R=r$ i.e. that $\left|z_{n}-\left(\frac{1}{2}+h i\right)\right| \geqq r$ where $h^{2}=r^{2}+k^{2}$. In fact,

$$
\begin{aligned}
\mid z_{n}- & \left.\left(\frac{1}{2}+h i\right)\right|^{2}-r^{2} \\
& =\left|z_{n+1}^{2}+c-\left(\frac{1}{2}+h i\right)\right|^{2}-r^{2} \\
& =\left|\left(\frac{1}{2}+R \cos \theta+i v\right)^{2}+\left(H^{2}-R^{2}+\frac{1}{4}\right)-\left(\frac{1}{2}+h i\right)\right|^{2}-r^{2} \\
& =|R(\cos \theta-2 v \sin \theta)+[R(\sin \theta+2 v \cos \theta)+H-h] i|^{2}-r^{2},
\end{aligned}
$$

substituting for $v^{2}$,

$$
\begin{aligned}
& =R^{2}(\cos \theta-2 v \sin \theta)^{2}+[R(\sin \theta+2 v \cos \theta)+H-h]^{2}-r^{2} \\
& =2 v\left[2 v R^{2}-(1+2 R \cos \theta)(h-H)\right],
\end{aligned}
$$

using $h^{2}-r^{2}=H^{2}-R^{2}$. The first factor is positive. The second rearranges to

$2 H R^{2}-(h-H)+2 R\left[R^{2} \sin \theta-(h-H) \cos \theta\right]$

$$
\geqq 2 H R^{2}-(h-H)-2 R\left[R^{4}+(h-H)^{2}\right]^{1 / 2},
$$

via the Schwartz inequality,

$$
=R^{2}(h+H)^{-1}\left\{2 H(h+H)-k^{2}-2 R\left[(h+H)^{2}+k^{4}\right]^{1 / 2}\right\}
$$

since $h-H=\left(h^{2}-H^{2}\right) /(h+H)=k^{2} R^{2} /(h+H)$. Further, $2 H(h+H)-k^{2} \geqq$ $3 k^{2}>0$ so positivity follows from

$$
\begin{array}{r}
{\left[2 H(h+H)-k^{2}\right]^{2}-4 R^{2}\left[(h+H)^{2}+k^{4}\right]} \\
=k^{2}\left(4 k^{2} R^{2}+4 H h+5 k^{2}\right)>0 .
\end{array}
$$

If $d\left(z_{0}, w_{2}\right)<\infty$, the convergence of $z$ to $w_{2}$ is identical to the convergence of $\bar{z}$ to $w_{1}$.

\section{REFERENCE}

1. C. S. Ogilvy, To what limits do complex iterated radicals converge?, Amer. Math. Monthly 77 (1970), 388-389.

Department of Mathematics, St. John's University, Jamaica, New York 11432 